\section{SAT0367 \\ PATIENTS HOSPITALISEDWITH GOUT AS MAIN DIAGNOSIS: COSTS AND EFFICIENCY ACCORDING TO HOSPITAL DEPARTMENTS}

M. Hernández Hurtado ${ }^{1}$, M. Á. Martínez Huedo ${ }^{2}$, A. Balsa Criado ${ }^{3}$, D. Peiteado López $^{3}$, E. De Miguel Mendieta ${ }^{3} .{ }^{1}$ Medical Student, Universidad Autónoma de Madrid, ${ }^{2}$ Teaching and investigation unit., Hospital Universitario La Paz;

${ }^{3}$ Rheumatology Department, Hospital Universitario La Paz-IdiPaz, Madrid, Spain

Background: Gout is the most common inflammatory articular disease in adults concerning a $1 \%-2 \%$ of the general population, and even a $4 \%-5 \%$ in older than 70 years. Recently, it has been reported an increase of the prevalence of gout, especially in developed countries.

Objectives: The main objective of this study is to describe the clinical and epidemiological characteristics of gout hospitalised patients in Spain (as the main reason of admission) and the economic outcomes of its management by the main care departments in charge when this disease is the principal diagnosis.

Methods: The main objective of this study is to describe the clinical and epidemiological characteristics of gout hospitalised patients in Spain (as the main reason of admission) and the economic outcomes of its management by the main care departments in charge when this disease is the principal diagnosis.

Results: From the whole 192.037 patients we have with gout diagnosis we performed a sub-analysis of 10.512 patients with gout as the main cause of hospitalisation, from witch the $85.9 \%$ are males. The admission number for this cause has remained constant or with a slight increase, with an average of 956 patients per year (equivalent to a $5.5 \%$ of gout total admissions). The average male age was 64.02 years (standard deviation (SD) 14.43) and 73.9 years (SD 13.69) for women $(p<0.001)$. When comparing patients with gout as main cause of admission against patients hospitalised by other causes, with gout as a secondary diagnosis. The former ones showed a significant lower percentage of comorbidities (type 2 diabetes, congestive heart failure, acute myocardial infarction and cerebrovascular disease).

Regarding the average of hospital stay of these patients, it was $6.71 \pm 6.8$ days with an average cost of $3471 \pm 2678 €$. Three medical specialities (Internal Medicine with 3852 hospital admissions (36.6\%), Rheumatology with $2600(24.7 \%)$ and Traumatology with $2033(19.3 \%))$, attended to an $80.6 \%$ of the total gout patients as main diagnosis for admission. It was noticed that the hospital stay was lower in the Traumatology Department with an average of $4.85 \pm 6.78$ days compared with the Rheumatology and Internal Medicine departments, which had an average of $6.52 \pm 5.65$ days and $7.76 \pm 6.83$ days respectively. However, it was found the lowest cost in the Rheumatology Department, with an average of 2892 $€$.

Abstract SAT0367 - Table 1. Average stay and costs by clinical departments: in gout as main diagnosis hospitalised patients.

\begin{tabular}{lccc}
\hline & $\begin{array}{l}\text { Internal } \\
\text { Medicin }\end{array}$ & Rheumatology & Traumatology \\
\hline $\begin{array}{l}\text { Number (\%) } \\
\text { Average hospital (SD) }\end{array}$ & $3852(36.6 \%)$ & $2600(24.7 \%)$ & $2033(19.3 \%)$ \\
days & $7.76(6.83)$ & $6.52(5.65)$ & $4.85(6.78)$ \\
Cost (SD) $€$ & $3549(1796)$ & $2892(1806)$ & $3715(2541)$ \\
\hline
\end{tabular}

$\mathrm{SD}=$ standard deviation

Conclusions: Only $24.7 \%$ of hospitalised patients with gout as main diagnosis are attended by Rheumatology Departments. However, Rheumatology was the most efficient clinical department in the care of this pathology. This conclusion should be considered in order to improve the management of gout in the health system

Disclosure of Interest: None declared

DOI: 10.1136/annrheumdis-2018-eular.7566

\section{SAT0368 ROLE OF JOINT ULTRASONOGRAPHY IN PATIENTS WITH GOUT STARTING TREATMENT WITH FEBUXOSTAT}

M. Novella-Navarro ${ }^{1}$, J.L. Cabrera-Alarcón ${ }^{2}$, E. Calvo-Aranda ${ }^{1}$, T. RodríguezAraya $^{1}$, J.-J. González-Martín ${ }^{1}$, P. Alcocer-Amores ${ }^{1}$, F. Aramburu-Muñoz ${ }^{1}$ C. Marín-Huertas ${ }^{1}$, A. Gómez-Gómez ${ }^{1}$, I. Amil-Casas ${ }^{1}$, C. Vergara-Dangond ${ }^{1}$, M. Valero-Expósito ${ }^{1}$, S. Rodríguez-Rubio ${ }^{1}$, P. García de la Peña- Lefebvre' ${ }^{1}$. ${ }^{1}$ Rheumatology, Hospital Universitario Madrid Norte Sanchinarro; ${ }^{2}$ Bionformatics, Molecular Biology Center, Severo Ochoa, Madrid, Spain

Background: Gout is the most prevalent arthritis globally, it is due to monosodium urate (MSU) crystals deposit on tissues, mainly in joints and periarticular structures. Although the main clinical pattern in gout is monoarticular, many patients suffer from oligo/polyarticular disease. Due to the information obtained through imaging tests such as ultrasonography (US), we know that the extent of MSU deposits are greater than we expected in many occasions, affecting clinically silent joints. Musculoskeletal US is a key tool due to its accessibility and safety, it determines accurately the current extent of deposits and joint involvement in gout, which may condition therapeutic changes.

Objectives: To evaluate the influence of articular US for clinical practice in Rheumatology when initiating treatment with febuxostat in patients with gout, determin ing the degree of crystalline deposit and articular ultrasonographic involvement.

Methods: Observational cross-sectional study of 129 patients diagnosed with gout (ACR criteria), treated with febuxostat (14 due to renal disease, adverse reaction/intolerance to allopurinol, and 115 due to non-response to allopurinol). One month after the onset of treatment, US examination was performed following the protocol proposed by Peiteado et $\mathrm{al}^{1}$, determining the number of joints with signs of gout (double contour, hyperechoic aggregates) and acute inflammatory activity (Doppler). Other variables were analysed: age, sex, hypertension, diabetes, dyslipidemia, chronic kidney disease, time of disease evolution and clinical pattern of joint involvement.

Results: 115 patients with febuxostat (112 men and 3 women), with a mean age of $57 \pm 13$ years and mean of disease evolution $14 \pm 10$ years. 59 patients had monoarticular clinical pattern, 46 oligoarticular and 10 polyarticular. US involvement: we observed acute inflammatory activity by Doppler in 47 patients $(40.86 \%)$, microcrystalline aggregates in 90 patients $(78.26 \%)$ and double contour sign in 53 patients $(42.08 \%)$. The mean uricemia at the time of the joint ultrasound examination was $7.4 \pm 1.8 \mathrm{~g} / \mathrm{dl}$. Of the 94 patients with uric acid levels $>6 \mathrm{mg} / \mathrm{dl}, 72$ presented extensive US involvement $(76.59 \%)$, whereas of the 21 patients with levels $<6 \mathrm{mg} / \mathrm{dl}$, US involvement was observed in 18 (85,71\%). From the observed variables, none was a risk predictor for joint involvement in binomial logistic regression model. Uricemia presented $\mathrm{OR}=0.83 \mathrm{Cl}(0.6-1.1)$

Conclusions: US quantification of MSU deposits can significantly condition the intensity of uricemia-reducing treatment regardless of serum uric acid levels. In this study, patients with non-target uricemia did not present a greater joint affection evaluated by US, however, those with $<6 \mathrm{mg} / \mathrm{dl}$, did present more affectation than might be expected. US examination of joints allows a more precise individu alization of the treatment in gout and should be incorporated to the periodic evaluation of these patients to optimise their prognosis

\section{REFERENCE:}

[1] Peiteado D, et al. Value of a short four-joint ultrasound test for gout diag nosis: a pilot study. Clin Exp Rheumatol 2012;30:830-7.

Disclosure of Interest: None declared

DOI: 10.1136/annrheumdis-2018-eular.1700

\section{SAT0369 COMPARISON OF RISK FACTORS FOR CARDIOVASCULAR DISEASES IN PTS WITH CPPD GOUT AND OSTEOARTHRITIS}

S. Vladimirov, M. Eliseev, O. Zhelyabina, M. Chikina. V. A. Nasonova Research Institute of Rheumatology, Moscow, Russian Federation

Background: The data on rates of comorbidities and risk factors for cardiovascular disease in CPPD is insufficient.

Objectives: To compare the rates of cardiovascular diseases, risk factors and the risk of death from cardiovascular disease in pts with CPPD, OA and gout. Methods: The study included 488 patients: 232 CPPD (111 m and $121 \mathrm{f}$ ) pts, 60 OA (19 $\mathrm{m}$ and $41 \mathrm{f}$ ) pts and 196 (169 $\mathrm{m}$ and $27 \mathrm{f}$ ) gout pts. The OA diagnosis was established based on ACR criteria, the CPPD diagnosis - on McCarty criteria and gout diagnosis - on the detection of monosodium urate crystals in synovia fluid. The mean age in CPPD pts was $57.6 \pm 18.2$ y., OA pts $-57.5 \pm 8.5$ y., and gout pts- $52.7 \pm 12.2$ y. Serum levels of cholesterol, glucose, uric acid, and hsCRP were obtained in all pts, and thorough evaluation for cardiovascular diseases was performedas well as including SCORE (Systematic COronary Risk Evaluation) scale assessment. Statistica 10.0, and Biostatistics were used for statistical data processing. Differences were considered to be statistically significant at $p<0.05$. Results: Obtained data are presented in table 1. High or very high risk of cardiovascular death (SCORE scale) was identified in $47.8 \%$ of CPPD pts, was comparable to that in gout pts $(48.9 \%)$ and exceeded the same in OA pts $(33 \%)(p<0.05)$ Higher probability of myocardial infarction (MI), chronic heart failure (CHF), chronic kidney disease stage $\geq 2$ (CKD) and higher mean serum CRP levels were established in CPPD and gout patients as compared to OA pts. There was no difference in rates of stroke, thrombosis, arterial hypertension $(\mathrm{AH})$, ischaemic dis ease (ICD), diabetes mellitus (DM 2), or in average glucose and cholesterol levels between CPPD, OA and gout pts. Average serum levels of uric acid were higher in patients with gout. 